\title{
DÜBLIN
}

Technological University Dublin

ARROW@TU Dublin

2008-01-01

\section{A Low Polarization Sensitivity All-fiber Wavelength Measurement System}

\author{
Ginu Rajan \\ Technological University Dublin, ginu.rajan@tudublin.ie \\ Yuliya Semenova \\ Technological University Dublin, yuliya.semenova@tudublin.ie \\ Gerald Farrell \\ Technological University Dublin, gerald.farrell@tudublin.ie
}

See next page for additional authors

Follow this and additional works at: https://arrow.tudublin.ie/engscheceart

Part of the Electrical and Computer Engineering Commons

\section{Recommended Citation}

Rajan, G., Semenova, Y., Farrell, G., Wang, Q., Wang, P.: A low polarization sensitivity all-fiber wavelength measurement system. IEEE Photonics Technology Letters, 2008, Vol.20, no. 17, pp.1464-1466.

doi:10.1109/LPT.2008.927899

This Article is brought to you for free and open access by the School of Electrical and Electronic Engineering at ARROW@TU Dublin. It has been accepted for inclusion in Articles by an authorized administrator of ARROW@TU Dublin. For more information, please contact arrow.admin@tudublin.ie, aisling.coyne@tudublin.ie, gerard.connolly@tudublin.ie.

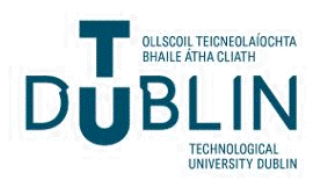




\section{Authors}

Ginu Rajan, Yuliya Semenova, Gerald Farrell, Qian Wang, and Pengfei Wang

This article is available at ARROW@TU Dublin: https://arrow.tudublin.ie/engscheceart/12 


\title{
A Low Polarization Sensitivity All-Fiber Wavelength Measurement System
}

\author{
Ginu Rajan, Yuliya Semenova, Gerald Farrell, Qian Wang, and Pengfei Wang
}

\begin{abstract}
A low polarization-sensitive all-fiber wavelength measurement system using a twisted macro-bend fiber edge filter together with polarization-insensitive (PI) 3-dB coupler is presented. Eliminating the polarization-dependent loss (PDL) is essential when macro-bending single-mode fiber is utilized as an edge filter. A new bending configuration to eliminate the PDL of a fiber edge filter is proposed in this letter by twisting the fiber by $90^{\circ}$ between two bending sections with equal length. The PDL of this configuration retains a low value even while the bending length is increased to achieve a steeper response for the edge filter. This configuration together with a PI 3-dB coupler makes the system to have very low polarization sensitivity and as a result high wavelength accuracy can be achieved.
\end{abstract}

Index Terms-Macro-bend fiber filter, polarization-dependent loss (PDL), twisted fiber filter, wavelength accuracy.

\section{INTRODUCTION}

A MACRO-BENDING single-mode fiber (SMF) can form the basis of an edge filter and can be utilized for ratiometric wavelength measurements [1]. Typically the macro-bend fiber filter and the 3-dB coupler in the ratiometric system are polarization-dependent devices. Our previous investigation on macro-bending standard SMF28 has shown that when the bending radius is relatively small, the polarization-dependent loss (PDL) is mainly caused by the fiber polymer coating layer and the PDL differs significantly for different bending radii [2]. If a system contains more than one polarization-sensitive device, its net PDL may fluctuate and hence the measured ratio also fluctuates and will lead to wavelength inaccuracy [3]. Prior to the introduction of systems demanding very high wavelength accuracy, the role of PDL in a ratiometric wavelength measurement system was not considered important. Thus, minimizing the polarization dependency of the components in the system is essential for high wavelength accuracy.

It is been proven earlier that PDL for a macro-bend fiber filter varies with bend radius. A minimum PDL filter can be obtained by choosing the correct bending radius corresponding to the minimal PDL; however, this requires a strict fabrication tolerance of the bending radius as PDL is highly sensitive to bend radius and varies drastically for a small change in bend radius. Furthermore, the inherent PDL will increase when the length of the bending section is increased, which is required to enhance the slope of spectrum for the edge filter. As an alternative to

Manuscript received February 26, 2008; revised May 15, 2008.

G. Rajan, Y. Semenova, G. Farrell, and P. Wang are with the Applied Optoelectronics Center, School of Electronic and Communications Engineering, Dublin Institute of Technology, Dublin 8, Ireland (e-mail: ginu.rajan@dit.ie).

Q. Wang is with the Data Storage Institute, Singapore 117608, Singapore.

Digital Object Identifier 10.1109/LPT.2008.927899 this straightforward method, in this letter, a new bending configuration is proposed to minimize the PDL. The proposed configuration contains two bending sections with equal length and with a $90^{\circ}$ twist in the middle. Based on this configuration, a minimum PDL is expected and this PDL is expected to be independent of the bending length involved. When used with a polarization-insensitive (PI) 3-dB coupler, the wavelength measurement system will be less dependent on the input polarization state and will give the highest accuracy.

\section{Method to Eliminate the Polarization DePendency OF A MACRO-BEND FIBER FILTER}

There are a couple of factors that result in polarization sensitivity for a bending fiber. It can originate from the anisotropy of the refractive index caused by the bending stress. Our recent investigation in [2] indicates that for SMF28 fiber with a relatively small bending radius (around $10 \mathrm{~cm}$ ), the polymer coating layer not only has a significant influence on the bend loss due to whispering gallery modes but also induces PDL. The theoretical model presented in [2] shows that the transverse-electric (TE) mode and transverse-magnetic (TM) mode propagating along the fiber suffer from different values of bend losses due to the different boundary conditions between the cladding and polymer coating layers for the two polarization states. The PDL of the fiber filter varies with wavelength as well as different bend radii. Thus, in the case of using a macro-bend fiber filter as an edge filter, the PDL of the filter introduces performance inaccuracy to the system, if there is a stress variation in the transmission fiber or a change of source to one other than the source used for calibration, which changes the polarization state of the input light. Therefore, it is necessary to eliminate the polarization dependency of the bend fiber edge filter to obtain high accuracy wavelength measurements. Since the PDL of the bend fiber originates from the difference in bend loss for TE and TM modes, one has to find a method to compensate this loss. One method to do this is by making two bending sections with equal length and by introducing a $90^{\circ}$ twist in the middle of the section. This changes the polarization state for the second bending section, i.e., the TE (TM) mode is turned to be the TM (TE) mode for the second bending section. The net effect is that the individual losses for the input TE and TM modes are equalized over the total length of the fiber so that the PDL can be minimized for the whole bending section. The schematic filter with and without a twist is shown in Figs. 1(a) and (b), respectively.

In our experiment, a standard SMF is used to build the filter. A bend radius of $10.5 \mathrm{~mm}$ with 15 bend turns is used as this gives suitable bend loss discrimination for a wavelength range of 1500-1580 nm without being affected by the signal-to-noise ratio of the source [1], [4]. A tunable laser with a wavelength range $1500-1600 \mathrm{~nm}$ is used as the source. A fiber polarization controller is used to change the input state of polarization of 


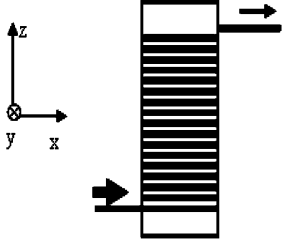

(a) (b)

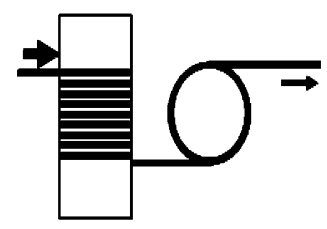

Fig. 1. Bending configurations of the macro-bend fiber filter. (a) Conventional bending. (b) Proposed configuration with a $90^{\circ}$ twist between the bending sections.

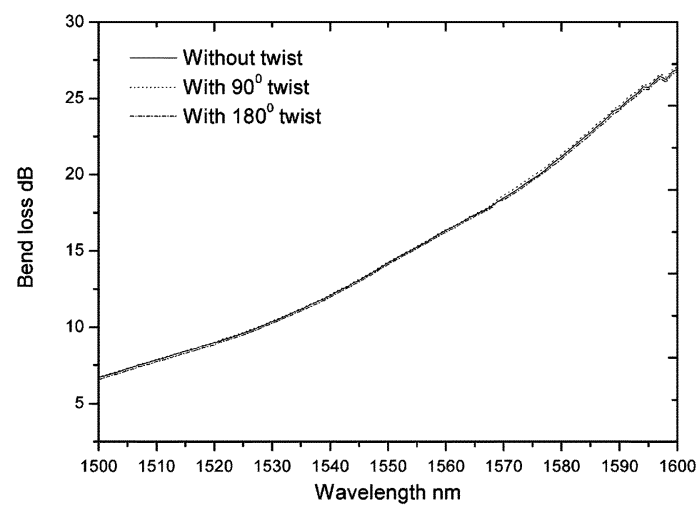

(a)

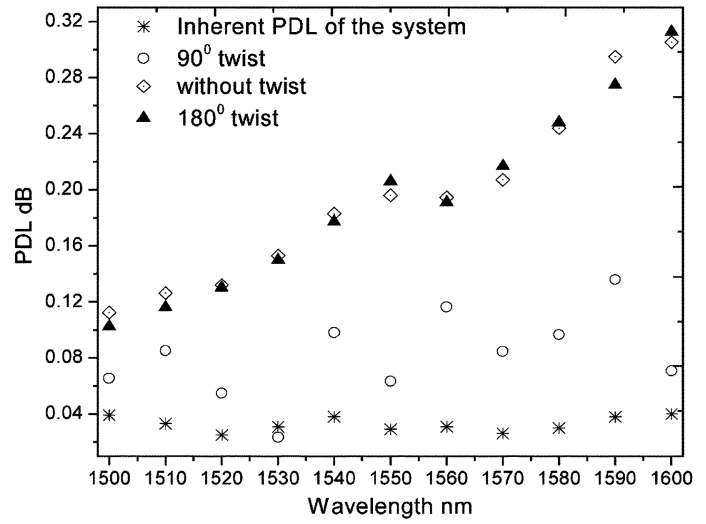

(b)

Fig. 2. (a) Measured bend loss and (b) PDL of the three configurations.

the signal and a dual-channel power meter employing InGaAs photodetectors is used to measure the power. Three configurations of the fiber filter were tested. A conventional fiber filter without a twist and filters with $90^{\circ}$ and $180^{\circ}$ twist in the middle of the fiber. For the second and third configurations, the fiber was wrapped with equal bend lengths (7.5 turns each) on two separate identical mandrels, with the second mandrel rotated by $90^{\circ}$ and $180^{\circ}$, respectively, to create a $90^{\circ}$ and $180^{\circ}$ twist in the middle of the two sections. The length of the fiber in between the two sections was approximately $2.5 \mathrm{~cm}$. The spectral response of the filter with three configurations is shown in Fig. 2(a). From the figure it is clear that the twist in the middle of the fiber has no significant influence on the bend loss response of the filter. However, the PDL shows a significant difference. The measured PDL for all three configurations for a wavelength range $1500-1600 \mathrm{~nm}$ at an interval of $10 \mathrm{~nm}$ is shown in Fig. 2(b). For the $180^{\circ}$ twist, the TE (TM) and TM (TE) modes return to

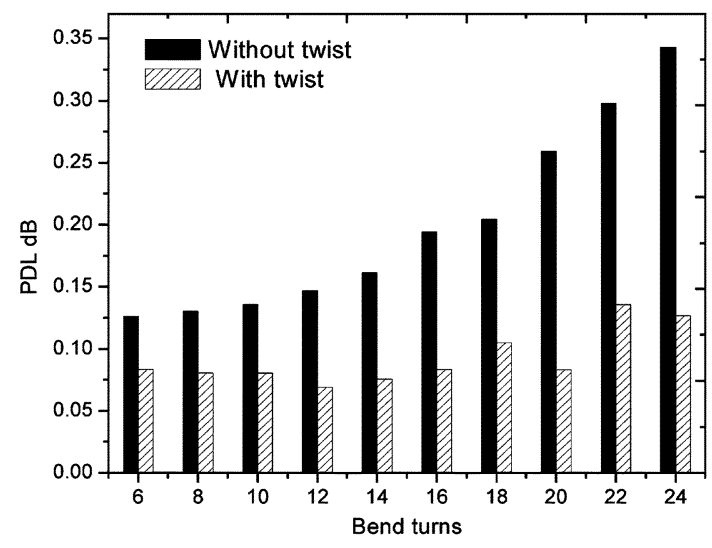

Fig. 3. Measured PDL of the fiber filter with twist and without twist.

their original state after the first bending section and such a filter shows the same PDL as the filter without twist. But for the filter with $90^{\circ}$ twist, after the first bending section TE (TM) mode is turned to TM (TE) and the individual losses for the input TE and TM modes are equalized and resulting in a low PDL. The polarization controller, the connection fibers, and the photodiodes used to measure the power also exhibit PDL. So the PDL of the system without the fiber edge filter is also measured and presented as the inherent PDL of the system. It is also known that the inherent PDL of the filter increases as the number of turns increases, which is necessary to get a steeper response. To see how the $90^{\circ}$ twist eliminates the PDL at higher bend lengths, the PDL of the filter is measured for different bend lengths and is shown in Fig. 3. A comparison of PDL for a fiber filter without a twist is also presented for the same number of turns.

PDL is not eliminated completely in the fiber filter due to physical inaccuracies such as small variations in the bend length of the two sections of the filter and variations in the twist angle of $90^{\circ}$ leading to residual PDL. The remaining PDL of the twisted configuration shown in Figs. 2(b) and 3 arises from these factors. It should be noted that a twist in the fiber induces circular birefringence to the fiber and can make it polarization-dependent. However, such stress-induced birefringence is very low in SMF28 fiber and this can be confirmed from Fig. 2(b), where the PDL of the filter with $180^{\circ}$ twist and a filter without a twist are closely matched which means that the twist-induced birefringence is negligible and its contribution to the PDL of the fiber filter is very small. Overall from the figures it is clear that the PDL of the fiber filter decreases considerably with a $90^{\circ}$ twist at higher bend lengths which in turn allows the filter to utilize a larger number of turns to obtain a steeper response and thus increase the measurement resolution of the system without reaching an unacceptable level of PDL.

\section{Low POLARIZATION-DEPENDENT WAVELENGTH MEASUREMENT SYSTEM}

A 3- $\mathrm{dB}$ coupler is one of the core components of any ratiometric wavelength measurement system. For a commercially available fused 3-dB coupler, it is known that it exhibits polarization dependency usually is in the order of $0.1 \mathrm{~dB}$ at $1550 \mathrm{~nm}$. In an all-fiber ratiometric system which consists of a macrobend fiber filter and 3-dB coupler, the PDL of the components leads to a ratio fluctuation and results in a very high wavelength 


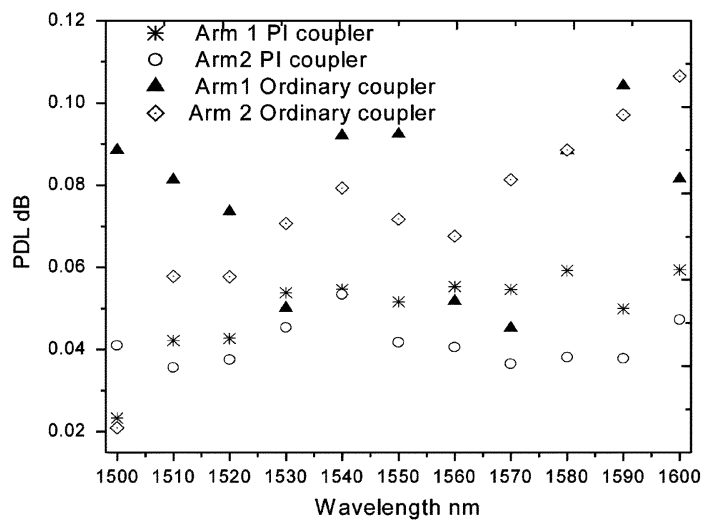

Fig. 4. Measured PDL of the arms a PI and an ordinary 3-dB coupler.

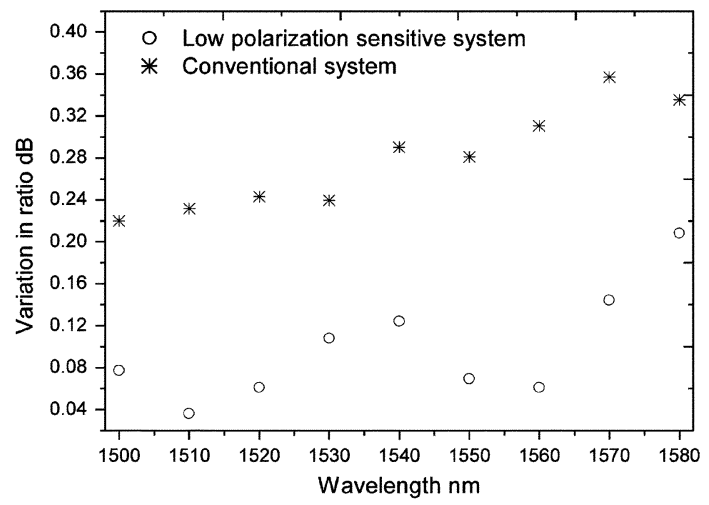

Fig. 5. Comparison of ratio errors in conventional system and low polarizationsensitive system.

error [5]. The PDL of the fiber filter can be eliminated by introducing a $90^{\circ}$ twist at the middle of the bending section as explained in the earlier section. But the PDL of the 3-dB coupler still will affect the system performance. One way to minimize the total polarization dependency of the system is the use of PI 3-dB couplers (couplers with very low PDL, in the range of 0.01-0.02 dB). For comparison the measured PDL of an ordinary 3-dB coupler and a PI 3-dB coupler is shown in Fig. 4. The measured PDL also includes the inherent PDL of the system. Thus, the combination of a PI coupler and a twisted macro-bend fiber filter can effectively reduce the total polarization dependency of the wavelength system.

A wavelength measurement system using a ratiometric scheme [1] has been built using a fiber edge filter with a $90^{\circ}$ twist and a PI coupler. A system based on a conventional fiber edge filter is also built. The number of bend turns used in both systems is 15 and the bend radius was $10.5 \mathrm{~mm}$. Both the systems were calibrated for a fixed polarization state. The input polarization state of the signal is changed using a polarization controller so it induces the maximum ratio and wavelength shift at a particular wavelength. The ratio and wavelength change from the calibrated value is measured for a wavelength range of $1500-1580 \mathrm{~nm}$ with an interval of $10 \mathrm{~nm}$ for both systems. The measured ratio and wavelength error are shown in Figs. 5 and 6, respectively. The measured wavelength errors are based on the local slope of the calibrated ratio response. The conventional system gives a wavelength error of $1.401 \mathrm{~nm}$ at 1550 , while

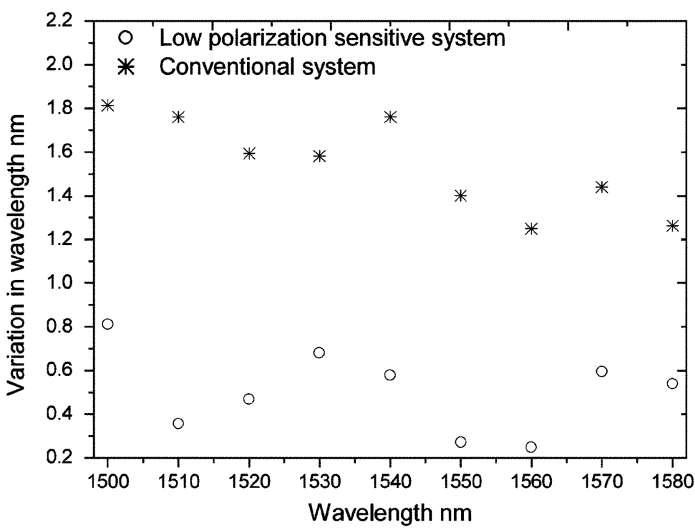

Fig. 6. Comparison of wavelength errors in conventional system and low polarization-sensitive system.

the low PDL system reduces the wavelength error to $0.272 \mathrm{~nm}$. From the figure it is clear, for the conventional system, PDL is a significant problem and induces a large wavelength error, but for the system with a twisted fiber filter and PI coupler, the wavelength error is effectively reduced which makes the system more accurate.

Thus for wavelength measurements based on macro-bend fiber filters the polarization dependency can be significantly reduced by the above proposed configuration and can deliver measurements with a high wavelength accuracy irrespective of the input state of polarization.

\section{CONClusion}

We have proposed and experimentally proved a new configuration for a macro-bend fiber filter to reduce its polarization dependency by introducing a $90^{\circ}$ twist in the middle of the bending section. In the twisted configuration, the number of turns of the filter can be increased to obtain a steeper response to increase the resolution of the system without a consequent increase in the PDL. A very low polarization-sensitive all-fiber wavelength measurement system using a twisted macro-bend fiber filter together with a PI 3-dB coupler was presented and verified experimentally. The wavelength error for the low polarizationdependent system was compared with that of a conventional fiber filter-based system and found to be significantly reduced. Thus, the proposed system can be used in cases where high accuracy is required, irrespective of the input polarization state.

\section{REFERENCES}

[1] Q. Wang, G. Farrell, T. Freir, G. Rajan, and P. Wang, "Low-cost wavelength measurement based on a macrobending single-mode fiber," Opt. Lett., vol. 31, pp. 1785-1787, 2006.

[2] Q. Wang, G. Rajan, P. Wang, and G. Farrell, "Polarization dependence of bend loss for a standard single mode fiber," Opt. Express, vol. 15, no. 8, pp. 4909-4920, 2007.

[3] N. Gisin, "The statistics of polarization dependent losses," Opt. Commun., vol. 114, pp. 399-405, 1995.

[4] Q. Wang, G. Farrell, and T. Freir, "Study of transmission response of edge filters employed in wavelength measurements," Appl. Opt., vol. 44, no. 36, pp. 7789-7792, 2005.

[5] G. Rajan, Q. Wang, Y. Semenova, G. Farrell, and P. Wang, "Effect of polarization dependent loss on the performance accuracy of a ratiometric wavelength measurement system," IET Optoelectron., vol. 2, no. 2 , pp. $63-68,2008$. 